\title{
Peso ao Nascer, Classe Social e Mortalidade Infantil em Ribeirão Preto, São Paulo
}

\author{
Birthweight, Social Class and Infant Mortality in Ribeirão Preto, São Paulo
}

\author{
Luis Eduardo A. de Almeida '; Marco Antonio Barbieri ${ }^{1}$; Uilho Antonio Gomes ${ }^{2}$; \\ Patricia Maria dos Reis ${ }^{1}$; Telma M. Chiaratti ${ }^{\text {; }}$ Valéria Vasconcelos ${ }^{1}$ \& \\ Heloisa Bettiol ${ }^{1}$
}

ALMEIDA, L. E. A. de; BARBIERI, M. A.; GOMES, U. A.; REIS, P. M. dos; CHIARATTI, T. M.; VASCONCELOS, V. \& BETTIOL, H. Birthweight, Social Class and Infant Mortality in Ribeirão Preto, São Paulo. Cad. Saúde Públ., Rio de Janeiro, 8 (2): 190-198, abr/jun, 1992. A study of infant mortality and its components was carried out in a cohort of live born children from hospitals of Ribeirão Preto, city of São Paulo, Brazil, whose mothers were interviewed shortly after deliverance.

Mortality was associated to social classes and birthweight.

The uneven distribution of mortality showed that social classes acted as distinct populations and that birthweight was not the only cause of this unballance.

Lower social classes were associated with higher low birthweight rates and mortality coefficients.

The study of infant mortality without taking into account the social differences may show coefficients that underestimate the magnitude of the problem among the poorest population segments.

Keywords: Birthweight; Social Class; Infant Mortality

\section{INTRODUÇÃO}

É evidente o interesse pelos fatores que vão agir na determinação de todas as categorias da mortalidade infantil, desde o início da gestação até o final do primeiro ano de vida.

Barreto (1948) dizia que a mortalidade infantil é o indicador mais sensível e importante do grau de desenvolvimento efetivo e também a medida do nivel cultural de qualquer sociedade. Na mesma época, Queiroz (1947) observou que a mortalidade deveria deixar de ser considerada problema exclusivo da Higiene para se

\footnotetext{
'Departamento de Puericultura e Pediatria da Faculdade de Medicina de Ribeirão Preto da Universidade de São Paulo. Avenida Bandeirantes, 3900, Ribeirão Preto, 14049-900, SP, Brasil.

2 Departamento de Medicina Social da Faculdade de Medicina de Ribeiräo Preto da Universidade de São Paulo. Avenida Bandeirantes, 3900, Ribeirão Preto, 14049-900, SP, Brasil.
}

tomar um grande capítulo da Economia e da Sociologia.

Sabe-se que há importantes diferenças dentro de uma população, o que faz com que uns experimentem uma melhor qualidade de vida, em contraste com outros que adoecem e morrem com mais frequiência, muitas vezes por nosologias também diferentes. Estas diferenças entre os indivíduos não se devem apenas às suas caracteristicas pessoais, inatas ou adquiridas, mas também, e primordialmente, ao modo como a sociedade se organiza e como se reparte a renda entre as classes. Por outro lado, é a situação social o contexto determinante na expressão das características pessoais (Bettiol, 1990).

Assim, a piora das condições biológicas da mãe e do concepto, relacionada a condições sócio-econômicas desfavoráveis, favorece maiores incidências de perdas fetais e de todas as outras categorias da mortalidade infantil.

De outra parte, assume grande importância o 
conhecimento do peso ao nascer para o estudo do desenvolvimento da criança e da mortalidade infantil. Essa importância advém, em grande parte, do fato de ser o peso medida antropométrica de fácil obtenção a nível populacional. Além disso, o peso reflete, de forma nítida, interferências às quais a mãe tenha sido submetida antes ou durante a gestação (Barros Filho, 1976).

Embora haja concordância que o baixo peso ao nascer se associe com um elevado número de óbitos (Chase, 1969; Lechtig et al., 1975; Peterson et al., 1978; Puffer \& Serrano, 1973), em alguns países (Cuba, China, Japão e Costa Rica, por exemplo) se reduziu a mortalidade infantil concomitantemente a uma estabilização na incidência de baixo peso ao nascer (Belizan et al., 1978; Monteiro, 1979; Solimano \& Galler, 1977). Um estudo recente, que procurou identificar as variáveis que mais contribuíram para explicar as diferenças interprovinciais observadas na mortalidade infantil em Cuba para cada ano compreendido entre 1977 e 1986, mostrou que o baixo peso ao nascer foi uma das que melhor explicou essas diferenças; contudo, pode-se estabelecer uma cadeia que identificou, em última instância, variáveis de matiz sócio-econômico - salário médio anual e saldo migratório interno - como explicativa destas diferenças (Perez \& León, 1990). Em estudo sobre peso ao nascer e mortalidade na infância, Monteiro (1979) concluiu que são principalmente as precárias condições de vida que recepcionam o recém-nascido, e não somente o peso ao nascer, que explicam o excesso de mortalidade infantil em São Paulo no período de estudo.

As diferenças observadas ao longo do tempo na intensidade do decréscimo da mortalidade perinatal são coerentes com as prioridades das políticas de saúde que, no final dos anos sessenta, impulsionaram o estabelecimento de centros de atenção perinatal de alta complexidade, conforme tem ocorrido em países como o Chile (Zaparero et al., 1989).

Outro exemplo foi o de Cuba após a Revolução de 1959 , quando as mudanças iniciadas nas condições de vida da população foram se refletindo, ao longo do tempo, em importantes avanços da saríte pública, levando a um pronunciado decréscimo da mortalidade infantil
(Bronfman \& Tuiran, 1984).

As observações anteriores foram corroboradas por Petterson et al. (1978), que, colocando em escala logarítmica os níves de peso ao nascer relacionados com a mortalidade neonatal nos EUA, em 1950, Califómia, e Ribeirão Preto, em 1968-70, observaram que as três curvas obedeciam à mesma conformação, isto é, altos coeficientes de mortalidade neonatal para baixo peso ao nascer; porém, as curvas se situavam em alturas diferentes, mostrando que recém-nascidos com baixo peso correm diferentes riscos em diferentes populações.

Este trabalho visa mostrar o comportamento dos coeficientes de mortalidade neonatal $e$ pós-neonatal e a mortalidade no primeiro ano de vida de uma coorte de crianças nascidas vivas em Ribeirão Preto, São Paulo, no período de um ano, segundo classe social e peso ao nascer.

\section{MATERIAL E MÉTODOS}

As informações que compõem basicamente todo o projeto desta investigação estão contidas nos 9067 questionários que representam, aproximadamente, $98 \%$ do universo de partos de crianças nascidas vivas nas oito matemidades dos hospitais de Ribeirão Preto, durante o período de um ano, e foram obtidas através de entrevistas com todas as puérperas que deram à luz entre $1^{2}$ de junho de 1978 e 31 de maio de 1979. Os questionários continham dados referentes aos pais, à renda, à gestação e ao parto (incluindo a atenção médica), ao recém-nascido (incluindo dados antropométricos colhidos ao nascer em todos os hospitais, e entre 12 e 24 horas de vida em dois hospitais), ao óbito da criança, quando ocorresse, à etnia e à reprodução humana. Maiores detalhes sobre a metodologia podem ser encontrados em publicação anterior (Barbieri et al., 1989).

Neste estudo, foram considerados os nascimentos únicos e óbitos apenas das crianças cujas mães eram procedentes de Ribeirāo Preto.

Para o estudo da mortalidade infantil, foi feito o levantamento dos óbitos das crianças incluidas no estudo até um ano após o encerramentio da coleta dos dados de nascimento. Foram utilizados os registros de óbito de toxke os 
cartórios do município de Ribeirão Preto, além dos mapas demógrafo-sanitários do Departamento Regional de Saúde e prontuários de todos os hospitais da cidade. Todas as declaraçōes de óbito das crianças menores de um ano nascidas no periodo do projeto foram selecionadas, copiadas integralmente e anexadas aos respectivos questionários. Foi verificado, também, que muitos nascidos vivos foram registrados como natimortos, quando o óbito ocorria logo após o nascimento.

A conceituação de classe social foi baseada no modelo proposto por Singer (1981), adaptado por Barros (1983) para uso epidemiológico, empregando indicadores que situam os individuos dentro das relaçōes sociais de produção, permitindo a discriminação das frações de classe na população estudada: a burguesia, proprietária dos meios de produção; a pequena burguesia, que trabalha mas é, ao mesmo tempo, proprietária dos seus meios de produção; o proletariado, que vende sua força de trabalho no mercado em troca de um salário e é composto pelo proletariado propriamente dito (trabalhadores do setor secundário) e subproletariado (desempregados, trabalhadores agrícolas e trabalhadores com ocupações irregulares, com inserção débil nas relações de produção); os sem classificação, representados pelas crianças nascidas de mães que se recusaram a responder - questionário por se encontrarem impossibilitadas física ou emocionalmente (perda do filho, por exemplo) ou por não compreenderem as perguntas ou o motivo das mesmas. Foram consideradas as seguintes categorias de peso ao nascer, segundo os critérios da Organização Mundial da Saúde (WHO, 1977); menor que 2500 gramas (baixo peso ao nascer) 2500 a 3000 gramas exclusive (peso insuficiente), e igual ou superior a 3000 gramas.

\section{RESULTADOS}

O total de óbitos das crianças nascidas de parto único, procedentes de Ribeirão Preto, foi 242, de um total de 6750 nascidos vivos também procedentes de Ribeirão Preto, o que correspondeu a um coeficiente de mortalidade infantil de 35,8 por 1000 nascidos vivos; os coeficientes de mortalidade neonatal precoce e neonatal tardia foram de 20,3 e 3,1 , respectivamente, o que dá um coeficiente neonatal de 23,4 e pós-neonatal de 12,4 por 1000 nascidos vivos.

As tabelas a seguir mostram a distribuição das crianças segundo a mortalidade (com os respectivos coeficientes e proporções) e classes sociais para peso ao nascer menor que $2500 \mathrm{~g}$ (Tabela 1), 2500 a 3000 gramas (Tabela 2), e igual ou superior a 3000 gramas (Tabela 3). $O$ total de crianças cujos pesos de nascimento eram desconhecidos foi 33 .

Observa-se que conforme aumenta o peso ao nascer, diminuem todos os coeficientes de todas as categorias de mortalidade e de todas as classes sociais.

Comparando-se os coeficientes obtidos com peso ao nascer de menos de $2500 \mathrm{~g}$ (Tabela 1) com os de $3000 \mathrm{~g}$ ou mais (Tabela 3 ), as maiores reduções se deram nos componentes neonatal precoce e tardio da mortalidade neonatal para todas as classes; porém, a redução do coeficiente neonatal precoce do proletariado foi 3,4 vezes menor que o da pequena burguesia e 1,8 vezes menor que o da burguesia. Em números, o proletariado teve reduzida em 18 vezes a mortalidade neonatal precoce quando o peso ao nascer passou de menos de $2500 \mathrm{~g}$ para $3000 \mathrm{~g}$ ou mais, a pequena burguesia reduziu 62 vezes e a burguesia, 33 vezes. O coeficiente de mortalidade neonatal tardio reduziu-se 11 vezes no proletariado e 90 vezes na burguesia, enquanto na pequena burguesia não houve nenhum óbito neonatal tardio nas faixas de peso consideradas. $\mathrm{Na}$ verdade, a grande redução ocorrida na burguesia significou 1 (um) óbito neonatal tardio da criança nascida com menos de $2500 \mathrm{~g}$ e nenhum óbito com peso ao nascer de $3000 \mathrm{~g}$ ou mais. No proletariado, a redução do coeficiente de mortalidade pós-neonatal (mais ligado a fatores econômico-sociais do que ao nascimento e à saúde perinatal) foi menor (três vezes), porém das crianças da pequena burguesia e da burguesia nascidas com menos de $2500 \mathrm{~g}$, não houve nenhuma que falecesse no periodo pós-neonatal, e apenas quatro crianças da pequena burguesia com peso de nascimento de $3000 \mathrm{~g}$ ou mais faleceram no mesmo periodo. A redução dos coeficientes de mortalidade infantil foi de 10 vezes no proletariado, 20 
TABELA 1 - Distribuição das Crianças Estudadas com Peso de Nascimento Inferior a 2500g, Segundo Tempo de Vida (Mortalidade) e Respectivos Coeficientes e Proporções e Classe Social

\begin{tabular}{|c|c|c|c|c|c|c|}
\hline \multirow{2}{*}{$\begin{array}{l}\text { Classe } \\
\text { Social }\end{array}$} & & \multicolumn{5}{|c|}{ Mortalidade } \\
\hline & & $\begin{array}{l}\text { Neonatal Precoce } \\
\text { (menor que } \\
7 \text { dias) }\end{array}$ & $\begin{array}{l}\text { Neonatal Tardia } \\
\text { (7-27 dias) }\end{array}$ & $\begin{array}{l}\text { Neonatal } \\
\text { (menor que } \\
28 \text { dias) }\end{array}$ & $\begin{array}{l}\text { Pós-neonatal } \\
\text { (28 dias a } \\
12 \text { meses) }\end{array}$ & $\begin{array}{l}\text { Infantil } \\
\text { (até } 12 \\
\text { meses) }\end{array}$ \\
\hline $\begin{array}{l}\text { Proletariado } \\
n=401\end{array}$ & $\begin{array}{c}N^{2} \\
\text { (Coefic.) } \\
\%\end{array}$ & $\begin{array}{c}54 \\
(134,7)\end{array}$ & $\begin{array}{c}7 \\
(17,5)\end{array}$ & $\begin{array}{c}61 \\
(152,2) \\
83,6\end{array}$ & $\begin{array}{c}12 \\
(29,9) \\
16,4\end{array}$ & $\begin{array}{c}73 \\
(182,1) \\
100\end{array}$ \\
\hline $\begin{array}{l}\text { Pequena } \\
\text { Burguesia } \\
n=23\end{array}$ & $\begin{array}{c}\mathrm{N}^{2} \\
\text { (Coefic.) } \\
\%\end{array}$ & $\begin{array}{c}5 \\
(217,4)\end{array}$ & $\begin{array}{c}0 \\
(0,0)\end{array}$ & $\begin{array}{c}5 \\
(217,4) \\
100\end{array}$ & $\begin{array}{c}0 \\
(0,0) \\
0\end{array}$ & $\begin{array}{c}5 \\
(217,4) \\
100\end{array}$ \\
\hline $\begin{array}{l}\text { Burguesia } \\
\mathrm{n}=11\end{array}$ & $\begin{array}{c}\mathrm{N}^{2} \\
\text { (Coefic.) } \\
\%\end{array}$ & $\begin{array}{c}1 \\
(90,9)\end{array}$ & $\begin{array}{c}1 \\
(90,9)\end{array}$ & $\begin{array}{c}2 \\
(181,8) \\
100\end{array}$ & $\begin{array}{c}0 \\
(0,0) \\
0\end{array}$ & $\begin{array}{c}2 \\
(181,8) \\
100\end{array}$ \\
\hline $\begin{array}{l}\text { Sem } \\
\text { Classificação } \\
n=48\end{array}$ & $\begin{array}{c}\mathbf{N}^{2} \\
\text { (Coefic.) } \\
\%\end{array}$ & $\begin{array}{c}13 \\
(270,8)\end{array}$ & $\begin{array}{c}2 \\
(41,6)\end{array}$ & $\begin{array}{c}15 \\
(312,4) \\
88,2\end{array}$ & $\begin{array}{c}2 \\
(41,6) \\
11,8\end{array}$ & $\begin{array}{c}17 \\
(354,0) \\
100\end{array}$ \\
\hline $\begin{array}{l}\text { Total } \\
n=483\end{array}$ & $\begin{array}{c}\mathbf{N}^{2} \\
\text { (Coefic.) } \\
\%\end{array}$ & $\begin{array}{c}73 \\
(151,1)\end{array}$ & $\begin{array}{c}10 \\
(20,7)\end{array}$ & $\begin{array}{c}83 \\
(171,8) \\
85,6\end{array}$ & $\begin{array}{c}14 \\
(28,9) \\
14,4\end{array}$ & $\begin{array}{c}97 \\
(200,8) \\
100\end{array}$ \\
\hline
\end{tabular}

TABELA 2 - Distribuiçảo das Crianças Estudadas com Peso de Nascimento entre $2500 \mathrm{~g}$ e $3000 \mathrm{~g}$ Segundo Tempo de Vida (Mortalidade) e Respectivos Coeficientes e Proporções e Classe Social

\begin{tabular}{|c|c|c|c|c|c|c|}
\hline \multirow{2}{*}{$\begin{array}{l}\text { Classe } \\
\text { Social }\end{array}$} & & \multicolumn{5}{|c|}{ Mortalidade } \\
\hline & & $\begin{array}{l}\text { Neonatal Precoce } \\
\text { (menor que } \\
7 \text { dias) }\end{array}$ & $\begin{array}{l}\text { Neonatal Tardia } \\
\text { (7-27 dias) }\end{array}$ & $\begin{array}{l}\text { Neonatal } \\
\text { (menor que } \\
28 \text { dias) }\end{array}$ & $\begin{array}{l}\text { Pós-neonatal } \\
\text { (28 dias a } \\
12 \text { meses) }\end{array}$ & $\begin{array}{l}\text { Infantil } \\
\text { (até } 12 \\
\text { meses) }\end{array}$ \\
\hline $\begin{array}{l}\text { Proletariado } \\
n=1104\end{array}$ & $\begin{array}{c}\mathbf{N}^{2} \\
\text { (Coefic.) } \\
\%\end{array}$ & $\begin{array}{c}11 \\
(10,0)\end{array}$ & $\begin{array}{c}1 \\
(0,9)\end{array}$ & $\begin{array}{c}12 \\
(10,9) \\
34,3\end{array}$ & $\begin{array}{c}23 \\
(20,8) \\
65,7\end{array}$ & $\begin{array}{c}35 \\
(31,7) \\
100\end{array}$ \\
\hline $\begin{array}{l}\text { Pequena } \\
\text { Burguesia } \\
n=128\end{array}$ & $\begin{array}{c}\mathrm{N}^{\mathrm{e}} \\
\text { (Coefic.) } \\
\%\end{array}$ & $\begin{array}{c}2 \\
(15,6)\end{array}$ & $\begin{array}{c}1 \\
(7,8)\end{array}$ & $\begin{array}{c}3 \\
(23,4) \\
100\end{array}$ & $\begin{array}{c}0 \\
(0,0) \\
0\end{array}$ & $\begin{array}{c}3 \\
(23,4) \\
100\end{array}$ \\
\hline $\begin{array}{l}\text { Burguesia } \\
n=79\end{array}$ & $\begin{array}{c}\mathrm{N}^{2} \\
\text { (Coefic.) } \\
\%\end{array}$ & $\begin{array}{c}0 \\
(0,0)\end{array}$ & $\begin{array}{c}0 \\
(0,0)\end{array}$ & $\begin{array}{c}0 \\
(0,0) \\
0\end{array}$ & $\begin{array}{c}1 \\
(12,7) \\
100\end{array}$ & $\begin{array}{c}1 \\
(12,7) \\
100\end{array}$ \\
\hline $\begin{array}{l}\text { Sem } \\
\text { Classificaçảo } \\
n=93\end{array}$ & $\begin{array}{c}\mathrm{N}^{2} \\
\text { (Coefic.) } \\
\%\end{array}$ & $\begin{array}{c}4 \\
(43,0)\end{array}$ & $\begin{array}{c}2 \\
(21,5)\end{array}$ & $\begin{array}{c}6 \\
(64,5) \\
66,7\end{array}$ & $\begin{array}{c}3 \\
(32,3) \\
33,3\end{array}$ & $\begin{array}{c}9 \\
(96,8) \\
100\end{array}$ \\
\hline $\begin{array}{l}\text { Total } \\
n=1404\end{array}$ & $\begin{array}{c}N^{2} \\
\text { (Coefic.) } \\
\%\end{array}$ & $\begin{array}{c}17 \\
(12,1)\end{array}$ & $\begin{array}{c}4 \\
(2,8)\end{array}$ & $\begin{array}{c}21 \\
(14,9) \\
43,7\end{array}$ & $\begin{array}{c}27 \\
(19,2) \\
56,3\end{array}$ & $\begin{array}{c}48 \\
(34,1) \\
100\end{array}$ \\
\hline
\end{tabular}


TABELA 3 - Distribuição das Crianças Estudadas com Peso de Nascimento acima de 3000g Segundo Tempo de Vida (Mortalidade) e Respectivos Coeficientes e Proporções e Classe Social

\begin{tabular}{|c|c|c|c|c|c|c|}
\hline \multirow{2}{*}{$\begin{array}{l}\text { Classe } \\
\text { Social }\end{array}$} & & \multicolumn{5}{|c|}{ Mortalidade } \\
\hline & & $\begin{array}{l}\text { Neonatal Precoce } \\
\text { (menor que } \\
7 \text { dias) }\end{array}$ & $\begin{array}{c}\text { Neonatal Tardia } \\
\text { (7-27 dias) }\end{array}$ & $\begin{array}{c}\text { Neonatal } \\
\text { (menor que } \\
28 \text { dias) }\end{array}$ & $\begin{array}{l}\text { Pós-neonatal } \\
\text { (28 dias a } \\
12 \text { meses) }\end{array}$ & $\begin{array}{l}\text { Infantil } \\
\text { (até } 12 \\
\text { meses) }\end{array}$ \\
\hline $\begin{array}{l}\text { Proletariado } \\
n=3642\end{array}$ & $\begin{array}{c}\mathrm{N}^{2} \\
\text { (Coefic.) } \\
\%\end{array}$ & $\begin{array}{c}27 \\
(7,4)\end{array}$ & $\begin{array}{c}6 \\
(1,6)\end{array}$ & $\begin{array}{c}33 \\
(9,0) \\
50,0\end{array}$ & $\begin{array}{c}33 \\
(9,0) \\
50,0\end{array}$ & $\begin{array}{c}66 \\
(18,0) \\
100\end{array}$ \\
\hline $\begin{array}{l}\text { Pequena } \\
\text { Burguesia } \\
\mathrm{n}=565\end{array}$ & $\begin{array}{c}\text { № } \\
\text { (Coefic.) } \\
\%\end{array}$ & $\begin{array}{c}2 \\
(3,5)\end{array}$ & $\begin{array}{c}0 \\
(0,0)\end{array}$ & $\begin{array}{c}2 \\
(3,5) \\
33,3\end{array}$ & $\begin{array}{c}4 \\
(7,1) \\
66,7\end{array}$ & $\begin{array}{c}6 \\
(10,6) \\
100\end{array}$ \\
\hline $\begin{array}{l}\text { Burguesia } \\
n=363\end{array}$ & $\begin{array}{c}\mathrm{N}^{2} \\
\text { (Coefic.) } \\
\%\end{array}$ & $\begin{array}{c}1 \\
(2,8)\end{array}$ & $\begin{array}{c}0 \\
(0,0)\end{array}$ & $\begin{array}{c}1 \\
(2,8) \\
100\end{array}$ & $\begin{array}{c}0 \\
(0,0) \\
0\end{array}$ & $\begin{array}{c}1 \\
(2,8) \\
100\end{array}$ \\
\hline $\begin{array}{l}\text { Sem } \\
\text { Classificação } \\
n=262\end{array}$ & $\begin{array}{c}\mathrm{N}^{2} \\
\text { (Cœefic.) } \\
\%\end{array}$ & $\begin{array}{c}5 \\
(19,1)\end{array}$ & $\begin{array}{c}1 \\
(3,8)\end{array}$ & $\begin{array}{c}6 \\
(22,9) \\
75,0\end{array}$ & $\begin{array}{c}2 \\
(7,6) \\
25,0\end{array}$ & $\begin{array}{c}8 \\
(30,5) \\
100\end{array}$ \\
\hline $\begin{array}{l}\text { Total } \\
n=4830\end{array}$ & $\begin{array}{c}\mathrm{N}^{\mathrm{e}} \\
\text { (Coefic.) } \\
\%\end{array}$ & $\begin{array}{c}35 \\
(7,2)\end{array}$ & $\begin{array}{c}7 \\
(1,4)\end{array}$ & $\begin{array}{c}842 \\
(8,6) \\
51,8\end{array}$ & $\begin{array}{c}39 \\
(8,1) \\
48,2\end{array}$ & $\begin{array}{c}81 \\
(16,7) \\
100\end{array}$ \\
\hline
\end{tabular}

vezes na pequena burguesia e 65 vezes na burguesia quando o peso ao nascer variou de menos de $2500 \mathrm{~g}$ a $3000 \mathrm{~g}$ ou mais.

Comparando-se os coeficientes de mortalidade infantil em todas as faixas de peso estudadas, o proletariado sempre apresentou maiores valores em relação às outras classes, com exceção da pequena burguesia na faixa de menos de $2500 \mathrm{~g}$, que apresentou o altissimo coeficiente de 217,4 por 1000 n.v., contra 182,1 do proletariado e 181,8 da burguesia. No entanto, o número de óbitos responsável por estes altos coeficientes foi baixo: cinco na pequena burguesia e dois na burguesia.

Houve um segmento social, representado pelas pessoas sem classificação quanto à classe social, que sempre apresentou os piores resultados em todas as comparações realizadas, sendo que o perfil epidemiológico deste segmento nos permite enquadrá-lo (em sua maioria) abaixo do proletariado.

A Figura 1 mostra bem as diferenças de coeficientes segundo o peso ao nascer e a classe social. O pequeno número de óbitos de crianças da pequena burguesia e da burguesia, qualquer que seja o peso ao nascer, se contrapōe aos altos valores do proletariado e dos sem classificaçãa. Para estes segmentos, a mortalidade infantil foi mais elevada que a das outras classes, independentemente do peso ao nascer, embora este, quando abaixo de $2500 \mathrm{~g}$, tenha correspondido, em todas as classes, a elevados coeficientes de mortalidade neonatal.

\section{DISCUSSÃO E CONCLUSÕES}

A mortalidade em uma população é função da freqüência com que ocorre a doença (incidencia) e a probabilidade de morte do doente (letalidade). A concepçāo biológica da morte, a nível individual, é insuficiente para explicar por si só a mortalidade considerada a nível coletivo. Saúde-doença é um processo que resulta do equilibrio entre o homem, fatores patogênicos externos distintos e os ambientes físico, biológico e social. Vários estudos têm demonstrado a relação entre a doença e a mcrte com variáveis como a renda familiar, a alimentação, o saneamento ambiental, a educação, a atenção médica, 
FIGURA 1 - Distribuição dos Coeficientes de Mortalidade Infantil (e seus Componentes) Segundo Peso e Classe Social

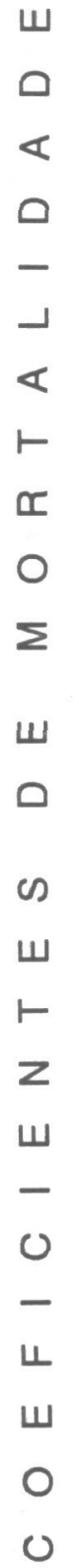


\begin{tabular}{|c|l|}
\hline$P$ & PROLETARIADO \\
\hline PB & PEQUENA BURGUESIA \\
\hline B & BURGUESIA \\
\hline SC & SEM CLASSIFICAÇÃO \\
\hline
\end{tabular}

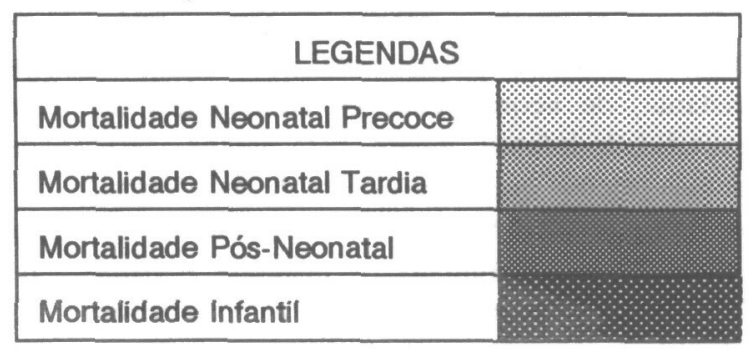


etc. Demonstram também que estes fatores estão estreitamente relacionados entre si, e a distribuição não-igualitária de todos esses componentes de vida é apenas a expressão visível de uma cadeia causal que tem origem na organização social, econômica e política (Behn, 1980).

Segundo Behn (1980), Taucher analisou a mortalidade infantil no Chile, em 1972-73, comparando os filhos de duas categorias de trabalhadores: aqueles inseridos no processo de produção de bens e em funções manuais - os operários - e os assalariados não-manuais situados sobretudo nos setores de serviços - os empregados de categoria média. Observou que a mortalidade entre os filhos dos operários foi o dobro da observada entre os filhos dos empregados médios, e este diferencial foi maior na mortalidade pós-neonatal (3,2 vezes). Dentro de cada grupo ocupacional, maior nível educacional esteve associado a menor mortalidade, porém entre os operários, um nível de educação acima da primária não se acompanhou de uma redução importante dessa taxa, sugerindo que o efeito da classe social foi de maior importância.

Por outro lado, Hale \& Druschell (1989) referem que o baixo peso ao nascer é o mais significante fator de risco para a mortalidade neonatal, enquanto a pobreza parece ser o maior determinante da mortalidade pós-neonatal.

Acontece, porém, que a pobreza é um dos fatores que geram os maiores índices de baixo peso ao nascer, ficando, portanto, o proletariado com os maiores coeficientes de mortalidade neonatal e pós-neonatal.

$O$ alto coeficiente de mortalidade infantil observado neste estudo na faixa de peso inferior a $2500 \mathrm{~g}$, da pequena burguesia, se concentrou exclusivamente no período neonatal, provavelmente representando crianças que nasceram muito mal e/ou portadoras de defeitos congênitos, incompatíveis com a vida extra-uterina.

Quando se estuda as proporções entre mortalidade neonatal e pós-neonatal, estas variam bastante, de acordo com os coeficientes de mortalidade infantil. Assim, para baixos coeficientes (menos que 25\%o), cerca de dois terços, ou aproximadamente $70 \%$ dos óbitos, são neonatais, principalmente na primeira semana de vida. Isto decorre do fato de a mortalidade infantil se resumir às anomalias congênitas e outras causas médicas de morte perinatal de difícil prevenção até o momento. Nestes casos, a diminuição dos coeficientes de mortalidade infantil se dá principalmente às custas da redução do componente pós-neonatal.

Para coeficientes moderados de mortalidade infantil (em torno de 50-100\%), o componente neonatal contribui com cerca de $40-50 \%$, quase metade, portanto, do valor total, e quando a mortalidade infantil é elevada (mais ou menos 100 a $150 \% 0$ ), este componente passa a contribuir com menor intensidade, em tomo de 30 a $35 \%$ do valor total, e o componente pós-neonatal, com os restantes $65-70 \%$. Quando os coeficientes de mortalidade infantil são muito elevados (mais ou menos em torno de 150-200\%o), as proporções entre neonatal e pós-neonatal giram em torno de $50 \%$ para cada uma; finalmente, com níveis excessivamente altos (acima de $220 \%$ ) o componente neonatal irá contribuir com cerca de $80 \%$ do total de mortes de menores de um ano. Nesta última situação, as condições ambientais são tão precárias, a assistência médica tão ausente, que diminuem sensivelmente as possibilidade de sobrevivência das crianças após o primeiro mês de vida (Almeida, 1988).

Os resultados obtidos no presente trabalho mostram que as proporções das mortalidades neonatal e pós-neonatal tiveram uma distribuição de acordo com os dados da literatura: para coeficientes muito altos ou muito baixos de mortalidade infantil, o componente neonatal tomou-se bem maior que o pós-neonatal; para coeficientes moderados, os componentes neonatal e pós-neonatal tiveram proporção semelhante.

De outro lado, o baixo peso ao nascer relacionou-se mais com a mortalidade neonatal precoce e tardia em todas as classes sociais consideradas. Conforme aumentou o peso ao nascer, diminuíram os coeficientes de todas as categorias de mortalidade em todas as classes sociais, porém as diminiriñes no proletariado foram muito menores que as da pequena burguesia e da burguesia.

Portanto, conclui-se que as classes sociais mais desfavorecidas ou os menores pesos ao nascer relacionaram-se sempre a maiores coeficientes de mortalidade ou se concentraram mais no período neonatal (quando os coefecientes 
foram muito elevados ou muito baixos) ou se distribuíram equiitativamente nos componentes neonatal e pós-neonatal (quando os coeficientes foram moderados), mostrando que os efeitos negativos do social estiveram e estão sempre presentes, desde o nascimento e ao longo de toda a curta vida de milhões de crianças no mundo inteiro.

\section{RESUMO}

ALMEIDA, L. E. A. de; BARBIERI, M. A.; GOMES, U. A.; REIS, P. M. dos;

CHIARATTI, T. M.; VASCONCELOS, V. \& BETTIOL, H. Peso ao Nascer, Classe Social e Mortalidade Infantil em Ribeirão Preto, São Paulo. Cad. Saúde Públ., Rio de Janeiro, 8 (2): 190-198, abr/jun, 1992.

Foi realizado um estudo sobre mortalidade neonatal e pós-neonatal e mortalidade no $1^{2}$ ano de vida de uma coorte de crianças nascidas vivas, de partos hospitalares em Ribeirão Preto, São Paulo, Brasil, cujas mães foram entrevistadas por ocasião do nascimento.

Relacionou-se mortalidade com classe social e peso ao nascer.

A distribuição desigual da mortalidade mostrou que as classes sociais se comportam como populações distintas, e o peso ao nascer não foi a causa isolada dessa desigualdade.

As classes sociais mais baixas corresponderam maiores taxas de baixo peso ao nascer e coeficientes de mortalidade mais elevados.

O estudo da mortalidade infantil, sem levar em conta a classe social, pode mostrar coeficientes que subestimam a magnitude do problema nas camadas mais pobres da população.

Palavras-Chave: Peso ao Nascer; Classe Social; Mortalidade Infantil

\section{REFERÊNCIAS BIBLIOGRAFICAS}

ALMEIDA, L. E. A., 1988. Mortalidade Infantil em Ribeirāo Preto. Estudo da Coorte Nascida entre Junho 78 e Maio 79. Análise de Algumas Variáveis Sociais e Biológicas. Tese de Mestrado, Ribeiråo Preto: Faculdade de Medicina de Ribeirão Preto, Universidade de São Paulo.
BARBIERI, M. A.; GOMES, U. A.; BARROS FILHO, A. A.; BETTIOL, H.; ALMEIDA, L. E. A. \& SILVA, A. A. M., 1989. Saúde perinatal em Ribeirāo Preto, SP, Brasil: a questąo do método. Cadernos de Saude Pública, 5: 376-387.

BARRETO, J. B., 1948. Tratado de Higiene. Vol. 1, $2^{\mathbf{l}}$ ed., Rio de Janeiro: Imprensa Nacional.

BARROS, M. A. B, 1983. Saide e Classe Social: Um Estudo Sobre Mortalidade e Consumo de Medicamentos. Tese de Doutorado, Ribeirão Preto: Faculdade de Medicina de Ribeirâo Preto, Universidade de Sảo Paulo.

BARROS FILHO, A. A., 1976. Influencia de Algumas Variáveis no Peso de Recém-Nascidos no Hospital das Clínicas de Ribeirão Preto. Tese de Mestrado, Ribeiråo Preto: Faculdade de Medicina de Ribeirão Preto, Universidade de S. Paulo.

BEHM, H., 1980. Determinantes económicas y sociales de la mortalidad en América Latina. Revista Cubana de Administración de Salud, 6: 1.30.

BELIZAN, J. M.; LECHTIG, A. \& VILLAR, J., 1978. Distribution of low birth weight babies in developing countries. American Journal of $O b-$ stetrics and Gynecology, 132: 704-706.

BETTIOL, H., 1990. Saúde Perinatal em Ribeirão Preto. Estudo de Algumas Variáveis Sociais e Biológicas no Perfil Reprodutivo de Mães Adolescentes. Tese de Mestrado, Ribeirảo Preto: Faculdade de Medicina de Ribeirão Preto, Universidade de São Paulo.

BRONFMAN, M. \& TUIRAN, R. A., 1984. La desigualdad social ante la muerte: classes sociales y mortalidad en la niñez. Cuadernos Médico-Sociales, 29/30: 53-75.

CHASE, H. C., 1969. Infant mortality and weigth at birth. 1960 - United States Birth Cohort. American Journal of Public Health, 59: 1618-1628.

HALE, C. B. \& DRUSCHELL, C. M., 1989. Infant mortality among moderately low birthweight infants in Alabama, 1980 to 1983. Pediatrics, 84: 285-289.

LECHTIG, A.; DELGADO, H.; MARTORELL, R.; BUND O. \& YARBROUGH, C., 1975. Effect of food suplementation during pregnancy on birthweight. Pediatrics, 56: 508-520.

MONTEIRO, C. A., 1979. O Peso ao Nascer no Município de São Paulo: Impacto sobre os Niveis de Mortalidade na Infancia. Tese de Doutorado, São Paulo: Faculdade de Saúde Pública, Universidade de São Paulo.

PEREZ, G. G. \& LEÓN, L. H., 1990. Desartolo social y mortalidad infantil, 1977-1986, Cuba. Un análisis regional. Revista de Sauide Pública, 24: 186-195. 
PETTERSON, R.; RENGSTRON, N.; STERKY, G. \& TAUBE, A., 1978. Birthweight distribution and socio-economic variables. Birthweight distribution as indicator of social development. In: SAREC REPORT (G. Storky \& L. Mellander), $\mathrm{n}^{\mathrm{q}}$ 2, pp. 33-39, II, Stockholm: Swedish Agency for Research Cooperation with Developing Countries.

PUFFER, R. R. \& SERRANO, C. V., 1973. Características de la mortalidad en la niñez. Publicación Cientifica $\mathfrak{n}^{\circ}$ 262, Washington: Organización Panamericana de la Salud.

QUEIROZ, J. S., 1947. Educação Sanitária. São Paulo: Divisão de Serviços da Saúde Escolar.

SINGER, P. I., 1981. Dominação e desigualdade: Estrutura de classe e repartição de renda no Brasil. Rio de Janeiro: Paz e Terra.

SOLIMANO, G. \& GALLER, J. R., 1977. Programas de nutrición materna: Un análisis crítica. Archivos Latino-Americanos de Nutrición, 27 (Suplemento 1): 223-240.

ZAPATERO, J. T.; CHAVEZ, R. T. \& TORRES, G. O., 1989. Mortalidad Perinatal en Chile, 1956-1986. Boletin de la Oficina Sanitária Panamericana, 106: 287-295.

WHO (World Health Organization), 1977. Recommended definiton, terminology and format for statistical tables related to the perinatal period and use of a new certificate for cause of perinatal deaths. Acta Obstetrica et Gynecologica Scandinavica, 56: 247-253. 